\title{
Comparison of Traction Controllers for Electric Vehicles with On-Board Drivetrains
}

\author{
Stefano De Pinto ${ }^{1}$, Christoforos Chatzikomis ${ }^{1}$, Aldo Sorniotti ${ }^{1, *}$, Member, IEEE, Giacomo Mantriota ${ }^{2}$ \\ ${ }^{1}$ University of Surrey, United Kingdom \\ ${ }^{2}$ Politecnico di Bari, Italy \\ *Corresponding author, a.sorniotti@ surrey.ac.uk, +44 (0)1483 689688
}

\begin{abstract}
An extensive literature discusses traction control system designs for electric vehicles. In general, the proposed control structures do not include consideration of the actuation dynamics, which are especially important for vehicles with onboard drivetrains, usually characterized by significant torsional dynamics of the half-shafts. This paper compares the performance of a selection of traction controllers from the literature, with that of PID and $H_{\infty}$ control structures specifically designed for on-board electric drivetrains. The analysis in the frequency domain and the simulation results in the time domain show the significant performance improvement provided by the control system designs considering the actuation dynamics.
\end{abstract}

Index Terms-Electric vehicle; traction control; on-board drivetrain; MTTE control; PID control; $H_{\infty}$ control; sliding mode control

\section{LIST OF SYMBOLS}

$A$ : system matrix of the state-space formulation of the plant $B$ : input matrix of the state-space formulation of the plant

$a_{2,2}, a_{3,3}$ : coefficients of state-space matrices

$b$ : coefficient of the first order sliding mode controller

$c_{\lambda}$ : longitudinal slip stiffness

$C$ : output matrix of the state-space formulation of the plant

$C_{\text {drag }}:$ aerodynamic drag coefficient

$e$ : error

$f_{0}$ : part of the rolling resistance coefficient independent of speed

$f_{2}$ : part of the rolling resistance coefficient to be multiplied by the square of speed

$f$ : coefficient of the first order sliding mode controller

$F:$ force

$G$ : transfer function

$G M$ : gain margin

$h$ : index used for defining the gain scheduling points

$H$ : matrix of the observer form implementation of the $H_{\infty}$ loop shaping controller

$i$ : gear ratio

$I$ : identity matrix

IACA: integral of the absolute value of the control action

$J$ : mass moment of inertia

$k$ : torsional stiffness

$K$ : gain
$L:$ wheelbase

$L_{\text {tire }}$ : tire relaxation length

$M$ : vehicle mass

$N$ : matrix of the observer form implementation of the $H_{\infty}$ loop shaping controller

$P$ : power

$P M:$ phase margin

$R$ : radius

$R M S E$ : root mean square of the error

$S$ : Laplace operator

$S$ : frontal area of the vehicle

$t:$ time

$T:$ torque

$T B$ : tracking bandwidth

$u$ : control input

$v, \dot{v}$ : vehicle speed, longitudinal vehicle acceleration

$V$ : Lyapunov function

$W$ : shaped plant in the $H_{\infty}$ observer form

$x$ : system states

$X$ : solution of the Riccati equation for $H_{\infty}$ control design

$y$ : output of the state-space formulation

$Z$ : solution of the Riccati equation for $H_{\infty}$ control design

$\alpha$ : parameter of the MTTE controller

$\beta$ : torsional damping coefficient

$\Delta T$ : torque correction

$\gamma$ : gain of the sliding mode controllers

$\Gamma$ : uncertain term, function of the states and time

$\epsilon$ : stability margin

$\eta$ : efficiency

$\theta, \dot{\theta}, \ddot{\theta}:$ angular position, speed, acceleration

$\lambda:$ slip ratio

$\mu$ : tire-road friction coefficient

$v$ : time derivative of the control input $u$

$\rho$ : air density

$\sigma$ : sliding variable

$\sigma^{*}$ : sliding variable of the second order sub-optimal sliding

mode controller

$\tau$ : time constant

$\varphi$ : parameter of the $H_{\infty}$ optimization procedure

$\phi$ : phase angle in the Bode plot

$\Phi$ : uncertain term, function of the states, input and time

$\chi$ : gain of the MTTE controller 
The following superscripts and subscripts are used in the paper:

act: actual

aer: aerodynamic

$A V C$ : active vibration controller

$d$ : delayed

$e q, 1 \ldots 5$ : equivalent terms of mass moment of inertia

$f, r$ : front, rear

fin: final

$g$ : gear

$H S$ : half-shaft

in: initial

$I$ : integral term

$m$ : motor

man: maneuver

$\max$ : maximum

MTTE: maximum transmissible torque estimation

$P$ : proportional term

ref: reference

res: motion resistance

roll: rolling resistance

$s, 1$ : primary shaft or first reduction stage of the transmission

$s, 2$ : secondary shaft or second reduction stage of the transmission

$S$ : shaped plant

$S M C$ : sliding mode controller

SOSM: sub-optimal sliding mode

$T C$ : traction controller

$v$ : vehicle

$w$ : wheel

$z$ : vertical direction

$\lambda$ : slip ratio

0 : linearization point

: estimated

$\sim$ : maximum

': corrected

\section{INTRODUCTION}

Electric vehicles (EVs) with multiple and individually controlled drivetrains present significant potential benefits with respect to more conventional EV layouts with a single electric drivetrain, an open differential and half-shafts. In fact, vehicle configurations with multiple motors have torquevectoring capability, i.e., yaw moment control is continuously actuated through the torque difference among the left and right wheels of the same axle. Torque-vectoring significantly improves vehicle cornering response, thus enhancing active safety. For example, torque-vectoring can increase the maximum lateral acceleration in steady-state conditions, and yaw damping in transient conditions [1]-[2].

Moreover, electric motor drives are characterized by more precise and fast torque response than internal combustion engines and hydraulic friction brakes. Hence, the performance of traction controllers (TC) and anti-lock braking systems (ABS) can be enhanced by the adoption of electric drivetrains. In particular, continuous feedback controllers for tracking a reference slip ratio can be implemented for both traction and braking.
An extensive academic literature describes TC systems, or more generally wheel slip controllers, potentially suitable for EVs with multiple drivetrains ([3]-[4]). The proposed solutions range from controllers not requiring the definition of a reference slip ratio ([5]-[7]), to controllers based on slip ratio feedback control. The feedback control action is achieved through gain scheduled proportional integral derivative (PID) and linear quadratic regulator (LQR) controllers ([8]-[10]), $H_{\infty}$ controllers [11], sliding mode controllers ([12]-[19]), and model predictive controllers ([20]-[21]).

Most of the industrial TC implementations, e.g., [22]-[23], adopt PID controllers with adaptable parameters, which are functions of the particular operating condition (such as the estimated tire-road friction coefficient and measured lateral acceleration). These controllers are commonly implemented on conventional vehicles with internal combustion engines and hydraulic friction brakes. Specific patents, such as [24], describe how to deal with particular situations, e.g., the case of a wheel in deep snow. The ABS algorithms on production vehicles are rule-based, and include a combination of decisions based on wheel acceleration and estimated longitudinal slip [25]. Recent patents, e.g., [26], describe wheel slip control strategies for electrified vehicles, including cooperation of the friction brakes and electric motors.

The continuous wheel slip controllers in the literature are based on the simplified equations of the wheel dynamics, with the slip ratio used as a state. They exclude consideration of: i) tire relaxation [27], which is very relevant to vehicle control [28]; and ii) drivetrain torsional dynamics, which can provoke a significant deviation between the expected and the actual wheel torque [29]. ii) is an important limitation, as the onboard (rather than the in-wheel) installation of the electric drivetrains is the preferred solution in many applications, mainly for packaging constraints.

In this respect, the novel contributions of the paper are:

- The analysis of the variation of the system frequency response, when considering tire relaxation and electric drivetrain torsional dynamics during TC design.

- The development of PID and $H_{\infty}$ controllers specifically designed for TC coupled with on-board electric drivetrains.

- The objective comparison of the performance of different control structures from the literature, with that of the traction controllers designed in this paper. Among the controllers from the literature, this paper focuses on PID control, two sliding mode control formulations, and the maximum transmissible torque estimation controller (MTTE).

The paper is structured as follows. Section III discusses the requirements for TC design. Section IV describes the models for control design and system simulation, including an example of experimental validation. The models are analyzed in the frequency domain in Section V. Section VI presents the TC formulations that are compared in Section VII. Finally, Section VIII reports the main conclusions.

\section{REQUIREMENTS AND CONTROL STRUCTURE}

Fig. 1 shows a typical longitudinal force $\left(F_{w}\right)-\operatorname{slip}$ ratio $(\lambda)$ characteristic of a passenger car tire, for different values of the 
tire-road friction coefficient, $\mu$. The value of $\lambda$ that maximizes $F_{w}$ varies with $\mu$. Stability control systems include some form of $\mu$-estimation (see [30]-[33]). However, these methods are usually very approximated, and in real-world applications $\mu$ is often estimated according to a few discrete levels. Also, for a given value of $\mu$, the shape of the $F_{w}(\lambda)$ characteristics can vary depending on the specific road and its condition, e.g., it can happen that $F_{w}$ is monotonically increasing with $\lambda$ (e.g., on snow), or in general that the slip ratio corresponding to the maximum value of $F_{w}$ is very different from the expected one. Moreover, the optimal value of $\lambda$ is a function of vertical tire load, $F_{z}$, which can be relatively easily estimated.

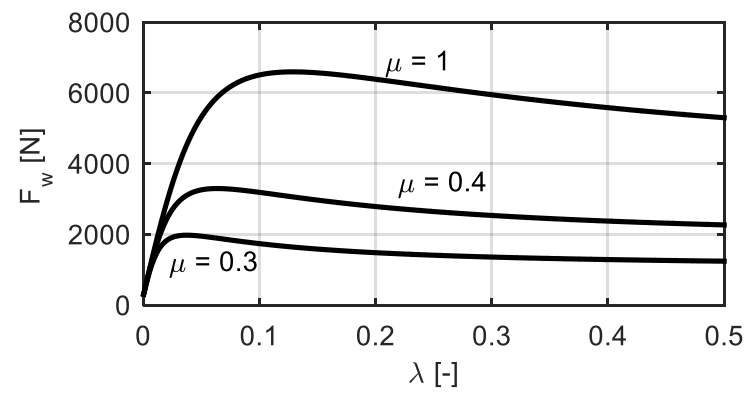

Fig. 1. Longitudinal tire force as function of the slip ratio for different values of the tire-road friction coefficient, for $F_{z}=6130 \mathrm{~N}$.

As a consequence, the first essential requirement of any TC is to be able to operate for a wide range of values of the local longitudinal slip stiffness, $c_{\lambda}$. This is defined as:

$$
c_{\lambda}=\left.\frac{\partial F_{w}}{\partial \lambda}\right|_{\lambda=\lambda_{\text {act }}}
$$

The second requirement is that the TC is operational only when strictly required, i.e., to limit $|\lambda|$. During its interventions, the TC outputs a reduction (and never an increase) of the electric motor torque with respect to the value produced by the low-level layer of the torque-vectoring controller. The TC must be de-activated when $\lambda$ is back within its normal values. Therefore, the control structure has to allow ease of activation/deactivation in a limited time frame.

Thirdly, the control action must be as smooth as possible, to allow comfortable vehicle operation, and not to provoke accelerated wear of the electric drivetrain hardware.

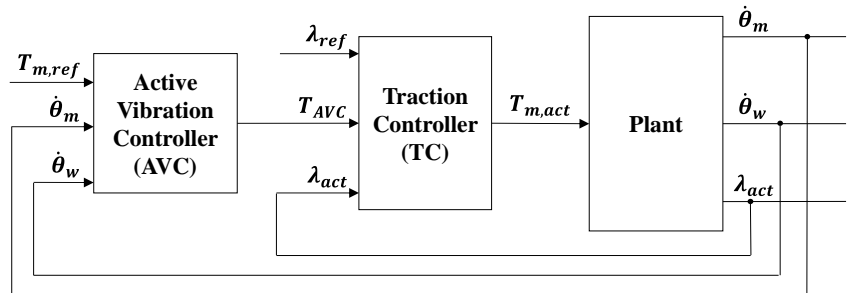

Fig. 2. Simplified schematic of a control structure including TC and AVC.

As shown in Fig. 2, the control problem is to regulate the estimated value of the longitudinal slip ratio, $\lambda_{\text {act }}$, to a setpoint, $\lambda_{\text {ref }}$, through the variation of the motor torque demand. However, the performance of on-board electric drivetrains is affected by their torsional dynamics, mainly caused by the half-shafts [29]. For improving drivability, an active vibration controller (AVC, which is a particular configuration of anti-jerk controller) is included in the control structure. The electric motor torque correction of the AVC is given by:

$$
\Delta T_{A V C}=-K_{A V C}\left(i_{g} \dot{\theta}_{m}-\dot{\theta}_{w}\right)
$$

The AVC behaves like a virtual damper with damping coefficient proportional to $K_{A V C}$, i.e., it is a gain-scheduled (with $v$ as a parameter) proportional controller of the torsional speed of the drivetrain (see [34] for details).

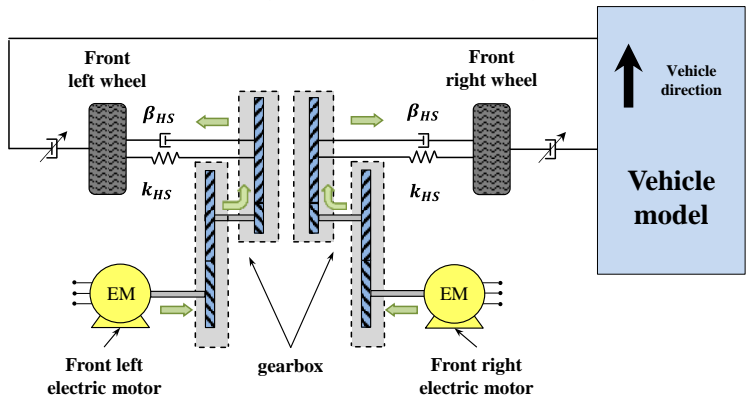

Fig. 3. Drivetrain layout of the case study FWD EV.

\section{MODELS FOR SLIP RATIO CONTROL}

The case study vehicle is a front-wheel-drive (FWD) EV with two on-board drivetrains (see Appendix A for the main parameters). Each drivetrain consists of an electric motor drive, connected to the respective wheel through a singlespeed transmission (with two stages of reduction) and a halfshaft with constant velocity joints (Fig. 3). The configuration with independent drivetrains on the two wheels of the same axle is discussed in the paper for simplicity, but the same theory is applicable to electric drivetrain architectures with a single on-board motor, a transmission and an open differential.

The control system design and assessment activity of this study is based on the following models at increasing levels of complexity:

- Model 1, including a steady-state linearized tire model and considering the rotating parts of the drivetrain as a rigid system. This is the model typology commonly adopted in the literature for wheel slip control system design (e.g., in [8], [13], [17]).

- Model 2, which, in addition to the features of Model 1, considers transient tire behavior through the concept of tire relaxation.

- Model 3, accounting also for the torsional dynamics of the half-shafts.

- Model 4, which adds the virtual damper of the AVC to the features of Model 3.

- Model 5, a non-linear simulation model in the time domain, implemented in Matlab-Simulink.

The following sub-sections report the main characteristics of the models. Models 1-4 are used for control system design in the frequency domain, while Model 5 is adopted for control system assessment in the time domain.

\section{A. Model 1}

A first order model is adopted for the electric motor drive dynamics:

$$
\tau_{m} \dot{T}_{m}+T_{m}=T_{m, r e f}
$$


Eqns. (4) and (5) express the rotational dynamics of the drivetrains and the equivalent longitudinal dynamics of the vehicle, modeled as an apparent mass moment of inertia:

$$
\begin{gathered}
T_{m}-T_{w, f} \frac{i_{g}}{\eta_{g}}-T_{\text {roll }, f} \frac{i_{g}}{\eta_{g}}=J_{e q, 1} \ddot{\theta}_{m} \\
2 T_{w, f}-2 T_{\text {roll }, r}-T_{\text {aer }}=J_{e q, 2} \ddot{\theta}_{v}
\end{gathered}
$$

where:

$$
\begin{gathered}
\eta_{g}=\eta_{s, 1} \eta_{s, 2}, \quad i_{g}=i_{s, 1} i_{s, 2} \\
J_{e q, 1}=J_{s, 1}+J_{m}+\frac{i_{s, 1}^{2}}{\eta_{s, 1}} J_{s, 2}+\frac{i_{g}^{2}}{\eta_{g}}\left(J_{w, f}+J_{H S}\right) \\
J_{e q, 2}=2 J_{w, r}+M R_{w}{ }^{2}
\end{gathered}
$$

According to a linear tire model, the tire-road traction torque, $T_{w, f}$, is a function of the longitudinal slip stiffness, $c_{\lambda}$, and the slip ratio, $\lambda$ :

$$
T_{w, f} \approx T_{w, f, 0}+c_{\lambda}\left(\lambda-\lambda_{0}\right) R_{w}
$$

where $\lambda$ is defined in Eq. (10) for traction conditions:

$$
\lambda=1-\frac{\dot{\theta}_{v}}{\dot{\theta}_{w}}=1-\frac{\dot{\theta}_{v}}{i_{g} \dot{\theta}_{m}}
$$

Eqns. (9) and (10) can be linearized as:

$$
T_{w, f} \approx T_{w, f, 0}+\frac{c_{\lambda} R_{w}}{i_{g} \dot{\theta}_{m, 0}}\left(\frac{\dot{\theta}_{v, 0}}{\dot{\theta}_{m, 0}} \dot{\theta}_{m}-\dot{\theta}_{v}\right)
$$

Linearizations are also used for the rolling resistance and aerodynamic drag torque contributions (reported in Appendix B). The model is expressed in state-space form with the following state and output vectors:

$$
\begin{gathered}
x=\left\{\dot{\theta}_{m}, \dot{\theta}_{v}, T_{m}\right\}^{T} \\
y=\left\{\dot{\theta}_{m}, \dot{\theta}_{v}, T_{m}, \lambda\right\}^{T}
\end{gathered}
$$

where the linearized slip ratio output is given by:

$$
\begin{aligned}
\lambda \approx & \lambda_{0}+\frac{\dot{\theta}_{v, 0}}{\dot{\theta}_{w, 0}^{2}} \dot{\theta}_{w}-\frac{1}{\dot{\theta}_{w, 0}} \dot{\theta}_{v} \\
& =\lambda_{0}+\frac{\dot{\theta}_{v, 0}}{\dot{\theta}_{w, 0}^{2}} i_{g} \dot{\theta}_{m}-\frac{1}{\dot{\theta}_{w, 0}} \dot{\theta}_{v}
\end{aligned}
$$

The design of feedback wheel slip controllers is based on the transfer function $G_{\lambda}(s)=\lambda(s) / T_{m, r e f}(s)$.

\section{B. Model 2}

In Model 2 the actual longitudinal traction force, $T_{d, w, f} / R_{w}$, is calculated from the steady-state traction torque, $T_{w, f}$ (Eq. (11)), corresponding to the current value of slip ratio, with the application of a relaxation length model for the evaluation of the transient effects, according to Eq. (14):

$$
\tau_{d} \dot{T}_{d, w, f}+T_{d, w, f}=T_{w, f}, \tau_{d}=\frac{L_{\text {tire }}}{\dot{\theta}_{w, 0} R_{w}}
$$

The resulting state variables and outputs are:

$$
\begin{gathered}
x=\left\{\dot{\theta}_{m}, \dot{\theta}_{v}, T_{m}, T_{d, w, f}\right\}^{T} \\
y=\left\{\dot{\theta}_{m}, \dot{\theta}_{v}, T_{m}, T_{d, w, f}, \lambda\right\}^{T}
\end{gathered}
$$

\section{Model 3}

Model 3 considers the dynamics of the half-shafts, modeled as combinations of torsion springs and dampers:

$$
T_{H S}=\beta_{H S}\left(i_{g} \dot{\theta}_{m}-\dot{\theta}_{w}\right)+k_{H S}\left(i_{g} \theta_{m}-\theta_{w}\right)
$$

The resulting states and outputs are:

$$
\begin{gathered}
x=\left\{\dot{\theta}_{m}, \dot{\theta}_{w}, \dot{\theta}_{v}, \theta_{m}, \theta_{w}, T_{m}, T_{d, w, f}\right\}^{T} \\
y=\left\{\dot{\theta}_{m}, \dot{\theta}_{w}, \dot{\theta}_{v}, \theta_{m}, \theta_{w}, T_{m}, T_{d, w, f}, \lambda\right\}^{T}
\end{gathered}
$$

\section{Model 4}

Model 4 includes the AVC, which is modeled as a variation of the reference motor torque, proportional to the torsional speed of the drivetrain:

$$
\begin{aligned}
\tau_{m} \dot{T}_{m} & +T_{m}=T_{m, r e f}+\Delta T_{A V C} \\
& =T_{m, r e f}-K_{A V C}\left(i_{g} \dot{\theta}_{m}-\dot{\theta}_{w}\right)
\end{aligned}
$$

The matrices $x, u, A$ and $B$ of the state-space formulation of Model 4 are reported in the Appendix C.

\section{E. Model 5}

To assess control system performance, a non-linear simulation model in the time domain was implemented in Matlab-Simulink. The main additional features with respect to Model 4 are: i) a non-linear tire model based on Pacejka magic formula [35]; ii) a transient tire model with relaxation length variable as a function of the operating conditions (e.g., $F_{z}$ and $\lambda$, according to the results in [27]); and iii) inclusion of the electric motor drive torque slew rate and windage losses.

An example of experimental validation of Model 5 is reported in Fig. 4, based on experimental longitudinal acceleration data from the vehicle demonstrator of the European Union funded E-VECTOORC project [36]. The specific experiments are electric motor torque sweep tests, with constant amplitude of the sinusoidal reference torque, and linearly increasing frequency. This reference torque profile allows the frequency response characterization of the longitudinal vehicle dynamics. The longitudinal tire parameters in Model 5 are the same as in [37] - [38]. The matching between the experimental and simulation results is particularly good, both with and without the AVC. The figure shows the significant attenuation of the drivetrain resonance provided by the AVC, without compromising drivetrain responsiveness.
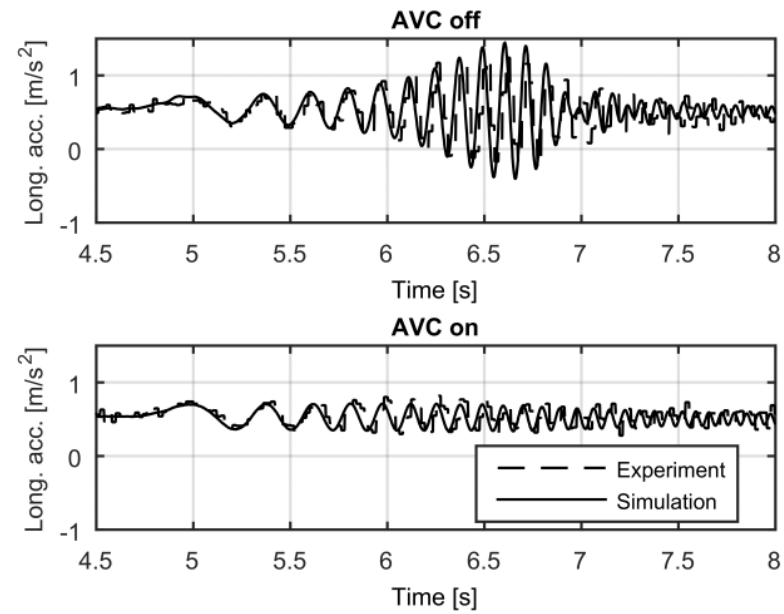

Fig. 4. Example of experimental validation of Model 5, with and without the AVC: time histories of longitudinal acceleration. 


\section{SYSTEM RESPONSE IN THE FREQUENCY DOMAIN}

This section critically analyzes the dynamic performance of Models 1-4 for the vehicle without TC. Under the assumption of $c_{\lambda}=1800 \mathrm{~N}$ (obtained from Pacejka model for $\lambda=0.033$, $\mu=0.3$ and $\left.F_{z}=6130 \mathrm{~N}\right)$, the Bode diagrams of $G_{\lambda}(\mathrm{s})=$ $\lambda(s) / T_{m, r e f}(s)$ are reported in Fig. 5. From Model 1 to Model 4 , the response of the system in the relevant frequency range changes from overdamped to underdamped, with a resonance peak in the region between $80 \mathrm{rad} / \mathrm{s}$ and $90 \mathrm{rad} / \mathrm{s}$. The frequency responses are also characterized by different corner frequencies.

Fig. 5 allows the first conclusion of this study, i.e., that Model 1, conventionally used for feedback tire slip control system design in the literature, is not suitable for the specific application of longitudinal slip control with on-board electric drivetrains. Model 3 and Model 4, including the torsional dynamics of the half-shafts, are required to this purpose. The relaxation dynamics are important as well $\left(L_{\text {tire }}=0.2 \mathrm{~m}\right.$ for the case study), as shown by the Bode diagram for Model 2, which substantially differs from that of Model 1. This is known especially by the industrial practitioners, even if the academic literature does not usually include tire relaxation in the models for wheel slip control system design. Moreover, Fig. 6, referring to Model 4, shows that the frequency response characteristics are functions of vehicle speed. This justifies the design of gain scheduled wheel slip controllers parameterized with $v$.
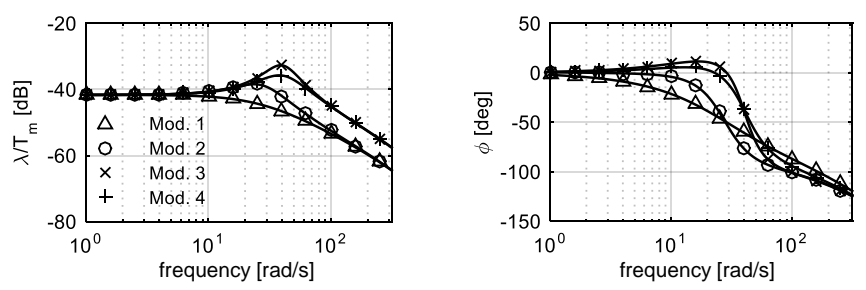

Fig. 5. Longitudinal slip ratio frequency response evaluated for Models 1-4 at $20 \mathrm{~km} / \mathrm{h}$.
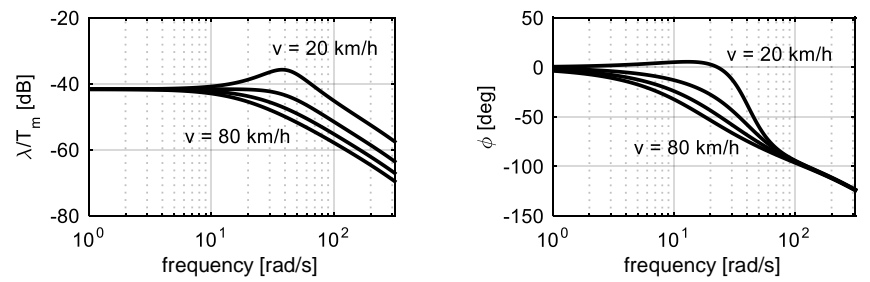

Fig. 6. Longitudinal slip ratio frequency response evaluated for Model 4 at vehicle speeds ranging from $20 \mathrm{~km} / \mathrm{h}$ to $80 \mathrm{~km} / \mathrm{h}$ with a step of $20 \mathrm{~km} / \mathrm{h}$.

\section{WHEEL SLIP CONTROL STRATEGIES}

Five slip ratio control approaches are considered:

- Maximum transmissible torque estimation (MTTE) control, as described in [6].

- First order sliding mode control (SMC), based on [13] and [42].

- Second order sub-optimal sliding mode (SOSM) control [13], [39]-[42].

- Proportional integral (PI) control with gain scheduling parameterized with $v$ [8].
- $H_{\infty}$ control based on loop shaping (the general theory of loop shaping is discussed in [43]-[45]), with gain scheduling with $v$.

The conditions for the activation and deactivation of the feedback slip controllers are:

$$
\text { Controller activation: } \lambda_{\text {act }}>\lambda_{\text {ref }}
$$

Controller de-activation: $\lambda_{\text {act }}<K \lambda_{\text {ref }}$, with $K<1$

The only exception to condition (19) is the MTTE controller, discussed in the following Section VI.A. Extensive driving cycle simulations with Model 5 were ran to verify the absence of undesired activations of the different TC algorithms during normal vehicle operation in high friction conditions.

\section{A. Maximum Transmissible Torque Estimation controller}

The MTTE was developed by Hori's research team [6], and experimentally demonstrated on EVs with in-wheel motors. The main benefit is that it avoids the problem of estimating vehicle velocity, which can be significant for four-wheel-drive vehicles, even if a longitudinal acceleration sensor is installed. For vehicles with only one driven axle, vehicle speed can be estimated for TC purposes from the angular speed measurement on the undriven wheels. Nevertheless, MTTE is an interesting TC option for the simplicity of its formulation.

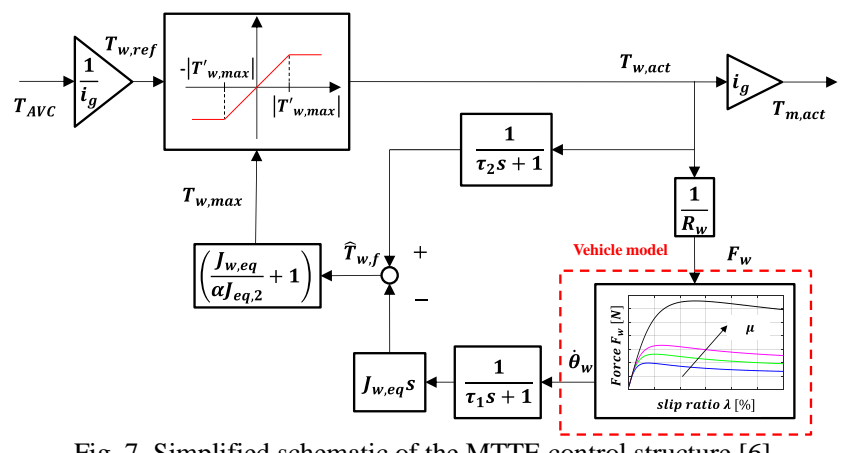

Fig. 7. Simplified schematic of the MTTE control structure [6].

According to MTTE, Eq. (20) estimates the torque corresponding to the longitudinal force between the tire and the road surface, from wheel acceleration and motor torque:

$$
\widehat{T}_{w, f}=\frac{\eta_{g} T_{m}}{i_{g}}-J_{w, e q} \ddot{\theta}_{w}
$$

where, in the specific case of on-board electric drivetrains, it is:

$$
J_{w, e q}=\frac{\eta_{g}\left(J_{s, 1}+J_{m}\right)}{i_{g}^{2}}+\frac{\eta_{s, 2} J_{s, 2}}{i_{s, 2}^{2}}+J_{w}+J_{H S}=\frac{J_{e q, 1}}{i_{g}^{2}}
$$

Eq. (21) neglects the drivetrain torsional dynamics.

An appropriate difference between chassis velocity and wheel velocity is necessary to provide the friction force of the tire. Accordingly, the ratio between the accelerations of the chassis and wheel can be described by the relaxation factor $\alpha$ :

$$
\alpha=\frac{\ddot{\theta}_{v}}{\ddot{\theta}_{w}}=\frac{\left(2 \widehat{T}_{w, f}-T_{r e s}\right) / J_{e q, 2}}{\left(2 T_{w, \text { max }}-2 \widehat{T}_{w, f}\right) / J_{w, e q}}
$$

where $2 T_{w, \max }$ indicates the drivetrain torque at the front wheels in limit conditions (hence the subscript ' $m a x$ '). By imposing $T_{\text {res }}=0$ (i.e., by neglecting $T_{\text {roll,r}}$ and $T_{\text {aer }}$ ) and rearranging Eq. (22), it is: 


$$
T_{w, \max }=\left(\frac{J_{w, e q}}{\alpha J_{e q, 2}}+1\right) \widehat{T}_{w, f}
$$

Since $\widehat{T}_{w, f}$ is available from Eq. (20), $T_{w, \max }$ can be calculated from Eq. (23), e.g., by considering a value of $\alpha=0.9$ [6].

In real applications, $T_{w, \max }$ can be smaller than $T_{w, \text { ref }}$ (i.e., the reference wheel torque according to the accelerator pedal position, vehicle speed and torque-vectoring controller output) not because of critical slip ratio conditions, but because of delays "at the acceleration start, which will cause a suddenly commanded acceleration to be temporarily constrained by $T_{w, \max }$ during acceleration phase" [6]. To avoid this problem, $T_{w, \max }$ is summed to the amplified (with gain $\chi$ ) value of $\dot{T}_{w, \text { ref }}$. The corrected maximum transmissible torque, $T_{w, \max }^{\prime}$, is then calculated as:

$$
T_{w, \text { max }}^{\prime}=T_{w, \text { max }}+\chi \dot{T}_{w, \text { ref }}
$$

$T_{w, \text { max }}^{\prime}$ is used to saturate $T_{w, \text { ref }}$ according to:

$$
\begin{aligned}
\text { if }-\left|T_{w, \max }^{\prime}\right|<T_{w, \text { ref }}<\left|T_{w, \max }^{\prime}\right| & \rightarrow T_{w, \text { act }}=T_{w, \text { ref }} \\
\text { if } T_{w, \text { ref }} \geq\left|T_{w, \max }^{\prime}\right| & \rightarrow T_{w, \text { act }}=\left|T_{w, \max }^{\prime}\right| \\
\text { if } T_{w, \text { ref }} \leq-\left|T_{w, \max }^{\prime}\right| & \rightarrow T_{w, \text { act }}=-\left|T_{w, \max }^{\prime}\right|
\end{aligned}
$$

The simplified block diagram of the MTTE controller is reported in Fig. 7.

\section{B. First order sliding mode controller}

The objective is to reach the sliding condition and remain on the sliding surface, defined by $\sigma_{\lambda}=0$ [42], where the sliding variable $\sigma_{\lambda}$ is:

$$
\sigma_{\lambda}=\lambda_{\text {act }}-\lambda_{\text {ref }}
$$

The control law consists of two terms: i) a term $F_{f} / b_{f}$, which would keep the system on the sliding surface, if the system dynamics were completely known; and ii) a switching term that ensures robustness with respect to modeling errors and disturbances [13], [39]-[41]. The control law is defined as follows [13]:

$$
T_{S M C}=\frac{1}{b_{f}}\left[-\gamma_{S M C} \operatorname{sat}\left(\sigma_{\lambda}\right)-f_{f}+\dot{\lambda}_{r e f}\right]
$$

where $F_{f}$ and $b_{f}$ are based on the rigid model of the drivetrain and are given by:

$$
\begin{gathered}
f_{f}=-\frac{\dot{v}}{i_{g} R_{w} \dot{\theta}_{m}}-\frac{v F_{w}}{J_{e q, 1} \eta_{g} \dot{\theta}_{m}^{2}} \\
b_{f}=\frac{v}{J_{e q, 1} R_{w} i_{g} \dot{\theta}_{m}^{2}}
\end{gathered}
$$

$\gamma_{S M C}\left(\sim 34 \mathrm{~s}^{-1}\right.$ for the simulations of this study) depends on the upper bound of the uncertainty. To guarantee that the closed loop system reaches $\sigma_{\lambda}=0$ in finite time, the Lyapunov function $V\left(\sigma_{\lambda}\right)=\frac{1}{2} \sigma_{\lambda}^{2}$ is considered. Hence, Lyapunov stability condition is derived:

$$
\begin{aligned}
\dot{V}\left(\sigma_{\lambda}\right)=\sigma_{\lambda} \dot{\sigma}_{\lambda}= & \sigma_{\lambda}\left(\dot{\lambda}_{\text {act }}-\dot{\lambda}_{r e f}\right) \\
& =\sigma_{\lambda}\left(-\frac{v}{\dot{\theta}_{m} i_{g} R_{w}}+\frac{v \ddot{\theta}_{m}}{\dot{\theta}_{m}^{2} i_{g} R_{w}}\right) \\
& \leq-\gamma_{S M C}\left|\sigma_{\lambda}\right|
\end{aligned}
$$

In practice, the required value of $\gamma_{S M C}$ can be calculated from the simulation results of the vehicle system in different operating conditions, through the re-arrangement of Eq. (30).

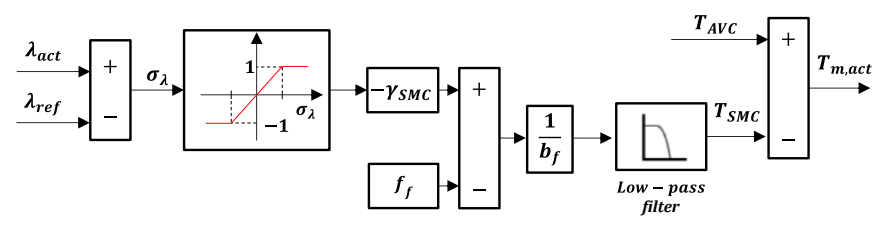

Fig. 8. First order sliding mode control structure, [13].

In the implementation of the controller, the saturation function in Eq. (27), coupled with a first order filter, is used to reduce chattering, which is detrimental to drivability and drivetrain durability. As a consequence, a pseudo-sliding mode, rather than an actual sliding mode, is induced in the system.

\section{Second order sub-optimal sliding mode controller}

The main advantage of second order SOSM control in its chattering avoidance formulation is the ability to achieve robustness with respect to matched disturbances, typical of sliding mode control, while avoiding control input chattering, which would compromise vehicle comfort and drivability [39]-[42]. In fact, with this SOSM formulation the discontinuity is on the time derivative of the control action, and not on the control action itself (see Fig. 9).

To apply second order SOSM control, the dynamic system must include a double integrator and have the following shape, with state variables $x_{1}(t)$ and $x_{2}(t)$ :

$$
\left\{\begin{array}{c}
\dot{x}_{1}(t)=x_{2}(t) \\
\dot{x}_{2}(t)=\Phi(x, u, t)+\Gamma(x, t) v(t)
\end{array}\right.
$$

In the anti-chattering formulation, $v(t)$ is the time derivative of the control action [39]-[41]. Moreover, in a neighborhood of the sliding manifold $\sigma_{\lambda}(x, t)=x_{1}(t)=0$, the uncertain terms $\Phi$ and $\Gamma$ must be bounded by known positive constants:

$$
\begin{gathered}
|\Phi(x, u, t)| \leq \widetilde{\Phi} \\
0<\tilde{\Gamma}_{1} \leq \Gamma(x, t) \leq \tilde{\Gamma}_{2}
\end{gathered}
$$

where $u(t)$ is the control input (i.e., the integral of $v(t)$ ).

For the specific system, Eq. (32) becomes:

$$
\left\{\begin{array}{r}
\dot{x}_{1}(t)=\dot{\sigma}_{\lambda}(x, t)=-\frac{\dot{v}}{\dot{\theta}_{m} i_{g} R_{w}}+ \\
\frac{v}{\dot{\theta}_{m}^{2} i_{g} R_{w}} \frac{T_{m}-\frac{i_{g}}{\eta_{g}} R_{w} F_{w}}{J_{e q, 1}} \\
\dot{x}_{2}(t)=\Phi(x, u, t)+\Gamma(x, t) \dot{T}_{m}(t)
\end{array}\right.
$$

The functions $\Phi(x, u, t)$ and $\Gamma(x, t)$ are reported in Appendix D. $v(t)$ is given by:

$$
\begin{aligned}
\dot{T}_{S O S M}=-\gamma_{S O S M} & \operatorname{sign}\left(\sigma_{\lambda}^{*}\right) \\
& =-\gamma_{S O S M} \operatorname{sign}\left(\sigma_{\lambda}-\frac{1}{2} \sigma_{\lambda, M}\right)
\end{aligned}
$$

To satisfy Lyapunov stability it must be:

$$
\gamma_{S O S M}>2 \widetilde{\Phi}
$$

The gain for the sliding mode controller was selected through test maneuvers simulated with Model 5, and the minimization of a cost function based on the combination of the performance indicators that will be defined in Section VII. In 
particular, for the results of this paper it is $\gamma_{S O S M}=3400$ $\mathrm{Nm} / \mathrm{s}$.

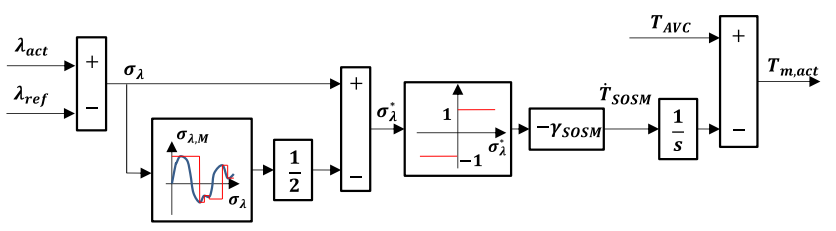

Fig. 9. Second order SOSM control structure.

\section{PI with gain scheduling}

For each $v$, the gains of the PI controller (see Fig. 10) were tuned by imposing: i) desirable gain margin $(G M)$ and phase margin $(P M)$ on the Bode diagram of the open-loop transfer function; and ii) desirable tracking bandwidth on the closedloop transfer function.

Multiple solutions are generally possible. For example, Fig. 11 plots the integral gain $\left(K_{I}\right)$ and the tracking bandwidth as functions of the proportional gain $\left(K_{P}\right)$, to achieve a gain margin of $\sim 15 \mathrm{~dB}$ and a phase margin of $\sim 60 \mathrm{deg}$, for $v=20$ $\mathrm{km} / \mathrm{h}$ and $v=60 \mathrm{~km} / \mathrm{h}$. Fig. 11 reports these loci for Models 1-4. In particular, Models 1 and 2, i.e., the models neglecting the half-shaft torsional dynamics, are characterized by a wide range of gain combinations providing the required stability and robustness properties, measured by $G M$ and $P M$. On the contrary, for Models 3 and 4 the range of $K_{P}$ and $K_{I}$ meeting the gain and phase margin specifications is very limited, and corresponds to a tracking bandwidth of $\sim 40-50 \mathrm{~Hz}$ at $-3 \mathrm{~dB}$.

\begin{tabular}{c|cc|cc|cc|cc}
\multicolumn{7}{c}{ Table I - Gain and phase margins: sensitivity analysis. } \\
\hline \hline $\begin{array}{c}\text { Speed } \\
{[\mathrm{km} / \mathrm{h}]}\end{array}$ & $\begin{array}{c}G M_{4,4} \\
{[\mathrm{~dB}]}\end{array}$ & $\begin{array}{c}P M_{4,4} \\
{[\mathrm{deg}]}\end{array}$ & $\begin{array}{c}G M_{4,1} \\
{[\mathrm{~dB}]}\end{array}$ & $\begin{array}{c}P M_{4,1} \\
{[\mathrm{deg}]}\end{array}$ & $\begin{array}{c}G M_{4,2} \\
{[\mathrm{~dB}]}\end{array}$ & $\begin{array}{c}P M_{4,2} \\
{[\mathrm{deg}]}\end{array}$ & $\begin{array}{c}\mathrm{GM}_{4,3} \\
{[\mathrm{~dB}]}\end{array}$ & $\begin{array}{c}\mathrm{PM}_{4,3} \\
{[\mathrm{deg}]}\end{array}$ \\
\hline \hline 20 & 14.9 & 59.9 & 8.6 & -17.5 & 8.1 & -16.9 & 16.0 & 63.5 \\
40 & 14.9 & 60.1 & 8.8 & -18.6 & 8.6 & -18.4 & 16.0 & 63.3 \\
60 & 15.1 & 60.0 & 8.6 & -18.4 & 8.6 & -18.9 & 16.0 & 63.1 \\
80 & 15.1 & 60.0 & 8.6 & -18.8 & 8.5 & -18.6 & 15.8 & 62.7 \\
\hline \hline
\end{tabular}

Table I reports the values of $G M$ and $P M$ evaluated with Model 4 (i.e., the most realistic model in the frequency domain), for the controllers designed through Models 1-4. Hence, the first subscript (i.e., '4' for all entries) of $G M$ and $P M$ in Table I indicates the model through which the margins are assessed, while the second subscript indicates the model through which the controller was designed. For Models 1 and 2, the selected values of $K_{P}$ and $K_{I}$ for the analysis in Table I are those providing similar tracking bandwidth to the gains obtained from Models 3 and 4.

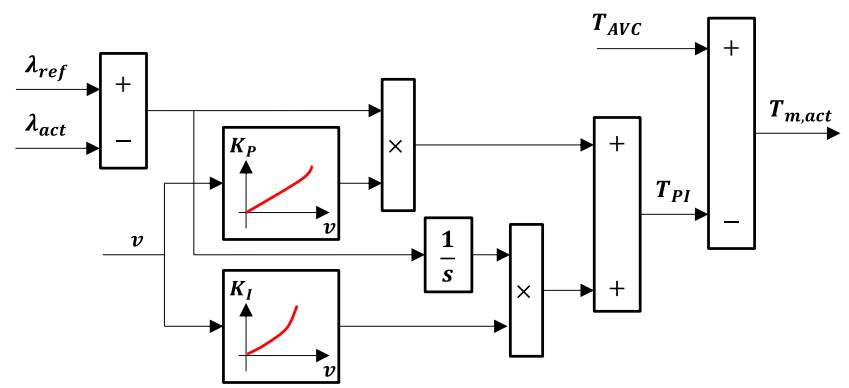

Fig. 10. Simplified schematic of the PI with gain scheduling control structure.

The important conclusion is that for the case study parameters the controllers based on Models 1 and 2 do not provide stability when assessed with the realistic model including drivetrain torsional dynamics, as the corresponding phase margins are negative.

The values of $K_{P}$ and $K_{I}$ providing $G M \sim 15 \mathrm{~dB}$ and $P M \sim 60$ deg have been computed for discretized values of vehicle speed along the relevant range of $v$, to obtain consistent system response. As a consequence, the resulting gains are scheduled as functions of $v$. Despite the significant variations of longitudinal tire slip stiffness during TC operation, the controller design was carried out for a fixed value of $c_{\lambda}$ for each $v$, as $c_{\lambda}$ variations are too fast to provide stable designs of gain scheduled controllers. Moreover, a gain scheduling on $c_{\lambda}$ would require the implementation of a longitudinal slip stiffness estimator, with potential issues in terms of accuracy and noise in real-world operating conditions. Nevertheless, the stability of the designed fixed-gain controllers with respect to $c_{\lambda}$ was verified for a wide range of longitudinal slip stiffness values.
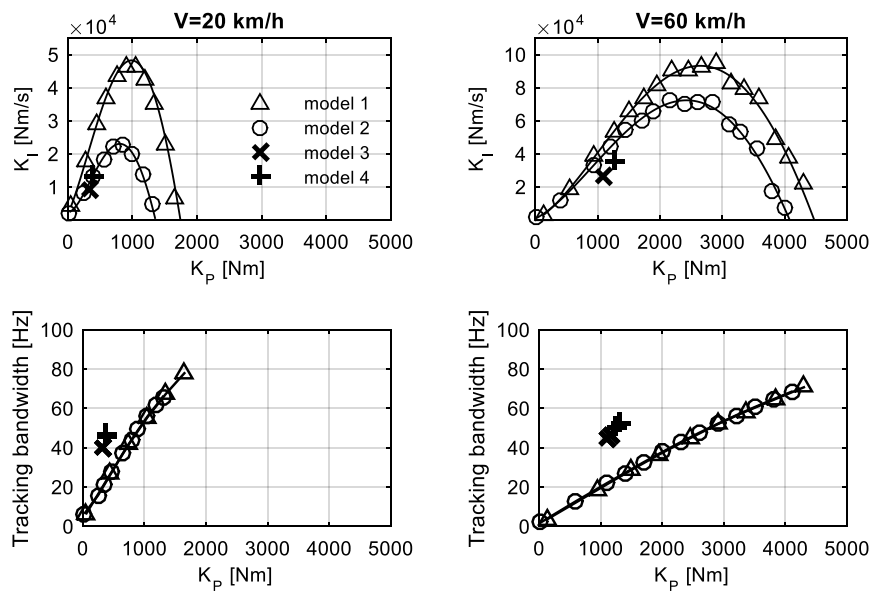

Fig. 11. Integral gain as function of proportional gain to achieve gain margins of $15 \pm 0.1 \mathrm{~dB}$ and phase margins of $60 \pm 0.1 \mathrm{deg}$.

\section{E. $H_{\infty}$ loop shaping controller with observer-based structure}

The mathematical formulation of the $H_{\infty}$ loop shaping robust stabilization problem is provided in [43]-[44]. $H_{\infty}$ loop shaping has been applied to many test cases in aerospace and vehicle engineering (see [45]-[46]), but not yet to the specific problem of TC for EVs. Following the analysis in Section VI.D, the plant $G_{\lambda}$ adopted for the control system design derives from Model 4 (i.e., the model including the AVC). For simplicity of notation, the dependence of the transfer functions on the Laplace operator $s$ is omitted in the remainder. An observer/state feedback structure of the $H_{\infty}$ loop shaping controller is employed to implement a gain scheduling scheme as a function of $v$. The $H_{\infty}$ loop shaping controller design procedure consists of the following steps:

i. Conversion of the transfer function $G_{\lambda}(s)$ into a state-space form:

$$
G_{\lambda}(v)=\left[\begin{array}{cc}
A(v) & B(v) \\
C(v) & 0
\end{array}\right]
$$

ii. Design of a pre-compensator, $W_{P I}$, with proportional and integral gains equal those used for the PI controller based on Model 4 in Section VI.D for the corresponding vehicle speeds. For the $H_{\infty}$ gain scheduling scheme, four speed values were selected, equal to $20 \mathrm{~km} / \mathrm{h}, 40 \mathrm{~km} / \mathrm{h}, 60 \mathrm{~km} / \mathrm{h}$ 
and $80 \mathrm{~km} / \mathrm{h}$. The transfer function of the shaped plant as a function of $v$ is:

$$
W(v)=W_{P I}(v) G_{\lambda}(v)=\left[\begin{array}{cc}
A_{s}(v) & B_{s}(v) \\
C_{s}(v) & 0
\end{array}\right]
$$

iii. Implementation of the $H_{\infty}$ loop shaping controller in the observer/state feedback form (Fig. 12):

$$
\left\{\begin{array}{c}
\dot{\hat{\lambda}}=\left[W(v)\left(\lambda_{\text {ref }}-\lambda_{\text {act }}\right)+\hat{\lambda}\left(A_{s}(v)+C_{s}(v)\right)\right] . \\
H_{S}(v)+B_{s}(v) T_{H_{\infty}} \\
T_{H_{\infty}}=N_{s}(v) \hat{\lambda}
\end{array}\right.
$$

where:

$$
\left\{\begin{array}{c}
H_{s}(v)=-Z_{s}^{T}(v) C_{s}^{T}(v) \\
N_{s}(v)=-B_{s}^{T}(v)\left(I-\varphi^{-2} I-\varphi^{-2} X_{s} Z_{s}\right)^{-1} X_{s}
\end{array}\right.
$$

$Z_{s}$ and $X_{s}$ are the solutions of the generalized algebraic Riccati equations of the $H_{\infty}$ loop shaping optimization. [44]. The order of the resulting compensator depends on the order of the system.

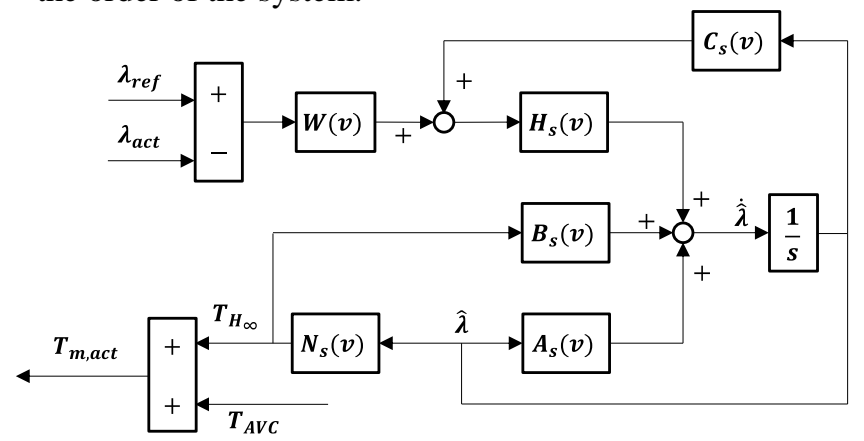

Fig. 12. $H_{\infty}$ loop shaping control structure.

iv. Implementation of the gain scheduling scheme. $H_{S}$ and $N_{s}$ can be scheduled by linear interpolation between the adjacent design points according to Eqns. (40)-(41), provided that the matrices $H_{S}$ and $N_{S}$ vary smoothly with vehicle speed, as it is the case here.

$$
\begin{aligned}
& N_{s}(v)=\frac{v_{h+1}-v}{v_{h+1}-v_{h}} N_{s}^{h}+\frac{v-v_{h}}{v_{h+1}-v_{h}} N_{s}^{h+1} \\
& H_{s}(v)=\frac{v_{h+1}-v}{v_{h+1}-v_{h}} H_{s}^{h}+\frac{v-v_{h}}{v_{h+1}-v_{h}} H_{s}^{h+1}
\end{aligned}
$$

Also the pre-compensator $W_{P I}(v)$ is scheduled by using linear interpolation between the two pre-compensators at adjacent design points $h$ and $h+1$, corresponding to the selected vehicles speeds.

Table II - Maximum stability margin, $\epsilon_{\max }$, for different vehicle speeds (evaluated with Model 4).

\begin{tabular}{c|cc|cc|cc|cc}
\hline \hline$v$ & \multicolumn{2}{|c|}{$\mathrm{PI}$} & \multicolumn{2}{c|}{$\mathrm{PI}_{80}$} & \multicolumn{2}{c|}{$H_{\infty}$} & \multicolumn{2}{c}{$H_{\infty, 80}$} \\
\cline { 2 - 8 }$[\mathrm{km} / \mathrm{h}]$ & $\epsilon_{\max }[-] T B[\mathrm{~Hz}]$ & $\epsilon_{\max }[-]$ & $T B[\mathrm{~Hz}]$ & $\epsilon_{\max }[-]$ & $T B[\mathrm{~Hz}]$ & $\epsilon_{\max }[-]$ & $T B[\mathrm{~Hz}]$ \\
\hline 20 & 0.32 & 46.2 & 0.01 & 152.5 & 0.53 & 20.2 & 0.18 & 62.0 \\
40 & 0.33 & 47.4 & 0.12 & 93.9 & 0.53 & 20.2 & 0.34 & 37.9 \\
60 & 0.33 & 50.5 & 0.24 & 68.5 & 0.52 & 20.8 & 0.45 & 27.6 \\
80 & 0.33 & 53.3 & 0.33 & 53.3 & 0.52 & 21.7 & 0.52 & 21.7 \\
\hline \hline
\end{tabular}

In Table II the robustness properties of the $H_{\infty}$ design are assessed through the maximum robust stability margin, $\epsilon_{\max }$, i.e., the maximum coprime uncertainty that can be tolerated before the system becomes unstable [44], for: i) the $H_{\infty}$ controller designed for $v=80 \quad \mathrm{~km} / \mathrm{h}, \quad$ i.e., $H_{\infty, 80}$ in the table; ii) the $H_{\infty}$ controller designed for each of the speeds, indicated as $H_{\infty}$ in the table; and iii) the same PI controllers used for the design of the $H_{\infty}$ controllers in i) and ii), respectively indicated as $\mathrm{PI}_{80}$ and $\mathrm{PI}$. The robustness benefit of the $H_{\infty}$ control designs with respect to the PI is evident. Also, the gain scheduling scheme provides consistent controller performance in terms of tracking bandwidth.

\section{PERFORMANCE ASSESSMENT}

The performance of the five TC formulations is assessed in three tip-in tests, simulated with Model 5. The time histories of the slip ratios $\left(\lambda_{\text {ref }}, \lambda_{\text {act }}\right)$ and motor torques $\left(T_{m, r e f}\right.$, $\left.T_{m, a c t}\right)$ are reported in Figs. 13-15. The maneuvers consist of a motor torque demand step, with amplitude of $180 \mathrm{Nm}$, starting from a vehicle speed of $30 \mathrm{~km} / \mathrm{h}$, for the following conditions:

- Maneuver 1: Tip-in test with reference slip ratio set to 0.033 for the controllers in Sections VI.B-VI.E (while the MTTE is not based on a reference slip ratio), and tire-road friction coefficient $\mu=0.3$. For the selected $\mu$, the slip ratio providing the maximum tire traction force is $\sim 0.05$, i.e., in this test $\lambda_{\text {ref }}$ is smaller than the slip ratio corresponding to the maximum tire force (see Fig. 1).

- Maneuver 2: Tip-in test with $\lambda_{\text {ref }}$ set to 0.10 and $\mu=0.3$. As a consequence, in this test $\lambda_{\text {ref }}$ is larger than the slip ratio corresponding to the maximum tire force (see Fig. 1).

- Maneuver 3: Tip-in test with $\lambda_{\text {ref }}=0.033$ and $\mu$ varying between 0.15 and 0.45 through a sequence of step variations with amplitude of 0.15 each (i.e., at $4 \mathrm{~s}$ with a $\mu$ variation from 0.30 to 0.15 for $1 \mathrm{~s}$, and at $7 \mathrm{~s}$ with a $\mu$ variation from 0.30 to 0.45 for $1 \mathrm{~s}$ ).

The purpose of Maneuvers 1-3 is to understand the robustness of the controller with respect to the variation of the longitudinal slip stiffness of the tires, which is associated to the respective $\lambda_{\text {ref }}$. The vehicle without TC would be subject to significant and persistent longitudinal slip values after the motor torque application of the tip-in tests. The performance of the MTTE is reported only in Fig. 13 and Fig. 15, as this controller does not explicitly track a reference slip ratio.

The performance of the TC formulations during Maneuvers 1-3 is assessed through the following indicators:

- The root-mean square value, $R M S E$, of the slip ratio error, $e(t)=\lambda_{a c t}-\lambda_{r e f}$, during the relevant part of the tests (i.e., after the tip-in applications):

$$
R M S E=\sqrt{\frac{1}{t_{\text {man,fin }}-t_{\text {man, in }}} \int_{t_{\text {man, in }}}^{t_{\text {man, fin }}}(e(t))^{2} d t}
$$

- $v_{f i n}$, i.e., the final value of vehicle speed at the completion of the test, since effective slip ratio tracking performance should be conjugated with high vehicle acceleration.

- The integral of the absolute value of the control action, IACA:

$$
I A C A=\frac{1}{t_{\text {man,fin }}-t_{\text {man, in }}} \int_{t_{\text {man, in }}}^{t_{\text {man, fin }}}\left|T_{T C}(t)\right| d t
$$

where $T_{T C}=u(t)$, i.e., $T_{T C}$ is the reference motor torque reduction imposed by the TC. This index is considered, as the expected slip ratio tracking performance should be delivered with limited control effort. 

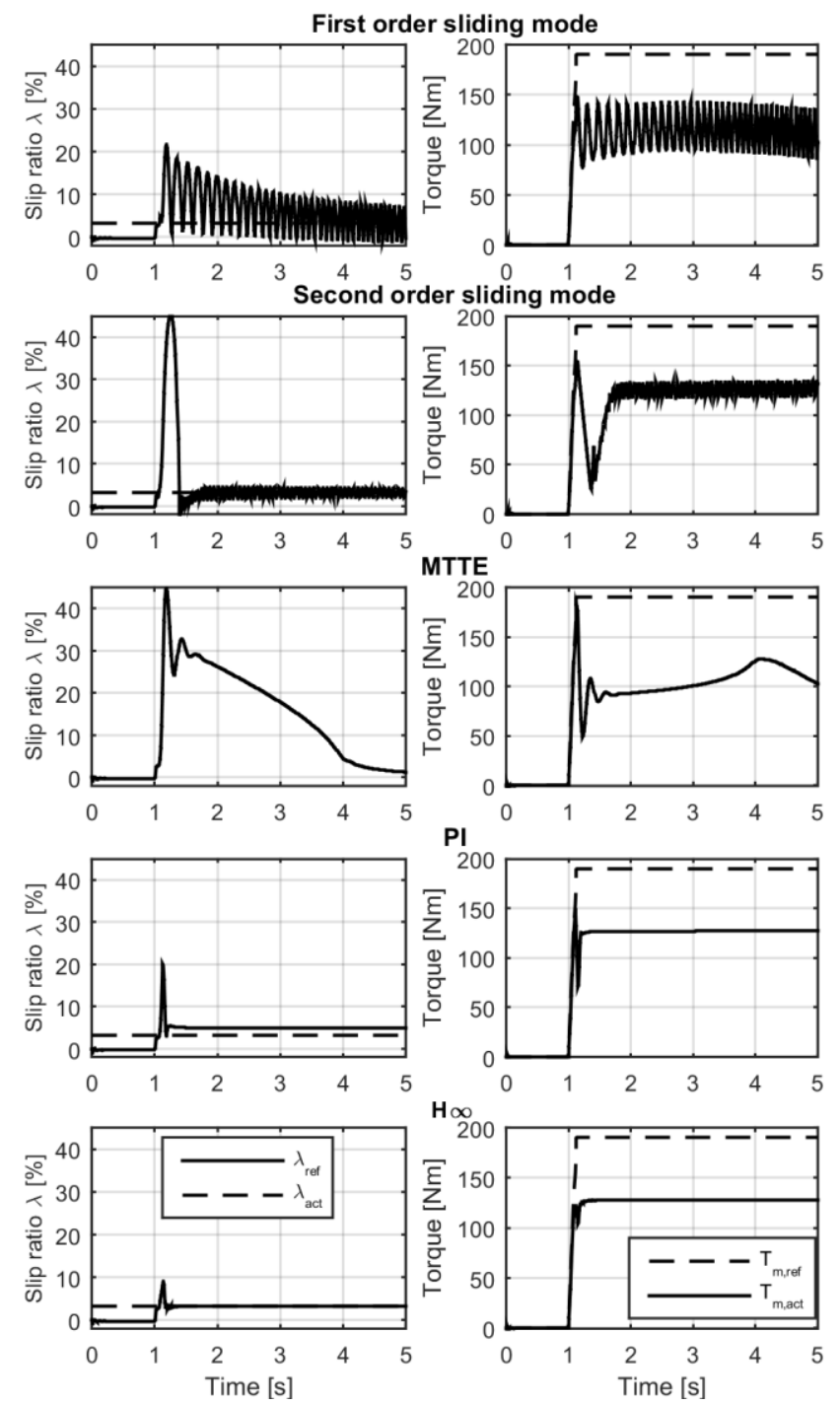

Fig. 13. Performance comparison of the controllers during Maneuver 1 (step torque demand, $\lambda_{\text {ref }}=0.033, v_{0}=30 \mathrm{~km} / \mathrm{h}, \mu=0.3$ ).

The analysis of Figs. 13-15 and Table III shows that:

- The first order sliding mode controller is characterized by significant chattering on $T_{S M C}$, which implies high values of $R M S E$ and IACA. The first peak of slip ratio, immediately after each transient, is relatively limited.

- The second order SOSM controller is characterized by very high slip ratio overshoots during and immediately after each transient of $T_{m, r e f}$ or $\mu$. On the other hand, the tracking performance in steady-state (or quasi-steady-state) conditions is relatively good.
- The MTTE is characterized by smooth torque demand and longitudinal slip profiles. However, a fixed value of the tuning parameter $\alpha$ can correspond to significant variations of the slip ratio during tests at constant $\mu$, which undermines the consistency of controller behavior. Nevertheless, this controller represents a viable solution especially for fourwheel-drive vehicles without advanced state estimators of vehicle speed.

- The PI and $\mathrm{H}_{\infty}$ controllers show similar performance in terms of RMSE, $v_{\text {fin }}$ and IACA. Their behavior is better in any aspect than that of the sliding mode controllers. A very marginal benefit in limiting the peak values of the slip ratio is achieved with the $\mathrm{H}_{\infty}$ controller (e.g., see the performance at $\sim 1.1 \mathrm{~s}$ in Fig. 13), with respect to the PI controller.
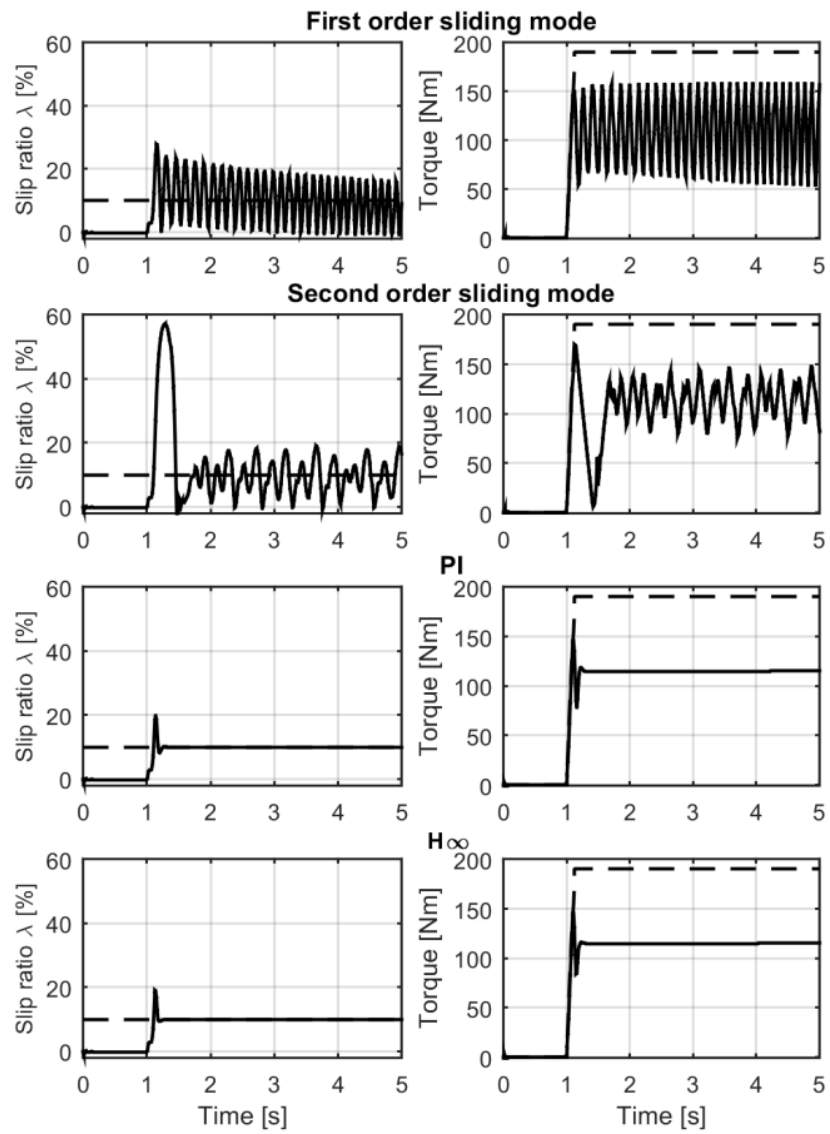

Fig. 14. Performance of the controllers during Maneuver 2 (step torque demand, $\lambda_{\text {ref }}=0.10, v_{0}=30 \mathrm{~km} / \mathrm{h}, \mu=0.3$ ).

Table III - Performance of the controllers evaluated through three maneuvers.

\begin{tabular}{|c|c|c|c|c|c|c|c|c|c|}
\hline \multicolumn{10}{|c|}{ Performance } \\
\hline Controllers & \multicolumn{3}{|c|}{ Maneuver 1} & \multicolumn{3}{|c|}{ Maneuver 2} & \multicolumn{3}{|c|}{ Maneuver 3} \\
\hline $1^{\text {st }}$ order SM & 0.0541 & 46.6 & 73.83 & 0.0784 & 45.7 & 78.67 & 0.0461 & 64.4 & 83.31 \\
\hline MTTE & - & 45.1 & - & - & 45.1 & - & - & 65.3 & - \\
\hline PI (Model 4) & 0.0171 & 48.8 & 61.02 & 0.0477 & 46.6 & 73.37 & 0.0134 & 71.7 & 63.81 \\
\hline$H_{\infty}($ Model 4) & 0.0168 & 48.8 & 60.96 & 0.0475 & 46.6 & 73.37 & 0.0137 & 71.7 & 63.84 \\
\hline
\end{tabular}



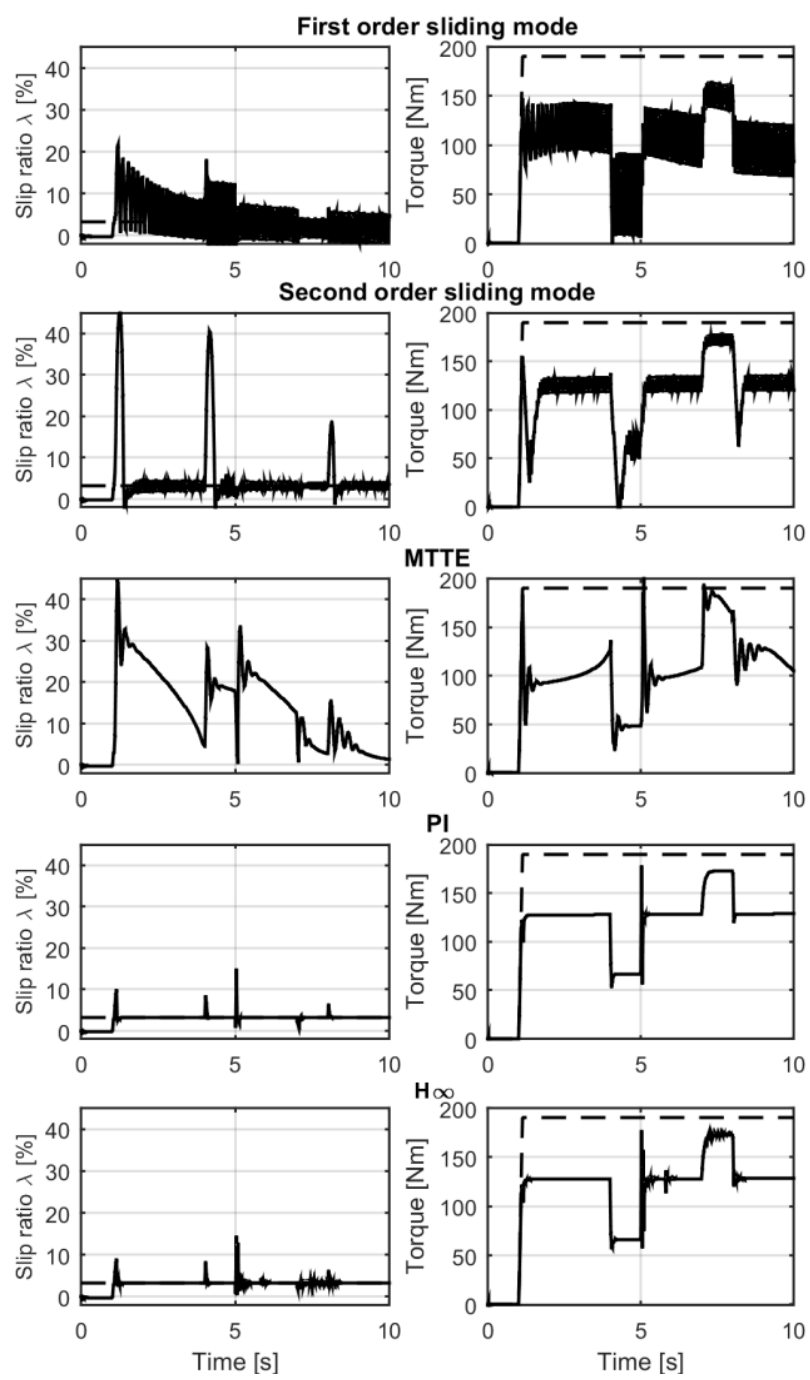

Fig. 15. Performance of the controllers during Maneuver 3 (step torque demand, $\lambda_{\text {ref }}=0.033, v_{0}=30 \mathrm{~km} / \mathrm{h}, \mu$ varying between 0.15 and 0.45 with steps of 0.15$)$.

\section{CONCLUSIONS}

The activity presented in this paper allows the following conclusions:

- The design of traction control systems for electric vehicles with on-board drivetrains should be based on models considering tire relaxation dynamics and, more importantly, drivetrain torsional dynamics.

- Controllers designed through the conventional simplified second order model (used in the literature) of wheel slip dynamics do not guarantee stability when applied to onboard electric drivetrain layouts with significant torsional dynamics. If torsional dynamics are considered, it is possible to obtain excellent tracking performance through longitudinal slip controllers based on continuous slip ratio control and relatively simple control structures.

- First order sliding mode control provides acceptable tracking performance, but despite the adoption of saturation functions and first order filters on the control action, this control structure provokes significant chattering. Based on the simulation results this controller does not appear to be actually viable for the specific application.

- Second order sliding mode guarantees good steady-state tracking performance. However, the peak values of slip ratio following fast variations of motor torque demand or tireroad friction coefficient are significant for the case study vehicle and maneuvers.

- The MTTE controller guarantees simplicity in the implementation and very smooth drivetrain torque profiles. However, constant values of its tuning parameters can correspond to very different values of the slip ratio during a maneuver executed at a fixed tire-road friction coefficient.

- Based on the selected objective performance indicators, the PI and $H_{\infty}$ controllers show the best behavior in terms of slip ratio tracking, longitudinal vehicle acceleration and reduced control effort. The high performance of these formulations is achieved through the inclusion of tire relaxation and drivetrain torsional dynamics in the models for control system design. This is the main conclusion of this study.

- Gain-scheduled PI and $H_{\infty}$ controllers are the recommended options for real vehicle implementation, as they combine relative simplicity of the control structure, good and predictable performance in different operating conditions, and ease of tuning. It is debatable whether the marginal performance benefit of the $H_{\infty}$ controller with respect to the PI controller is worth the increased implementation effort.

Future developments will be aimed at the experimental validation of the findings of these simulation-based results.

\section{ACKNOWLEDGMENT}

The research leading to these results has received funding from the European Union Seventh Framework Programme FP7/2007-2013 under grant agreements no. 605502 (PLUSMOBY), no. 608784 (FREE-MOBY) and no. 284708 (E-VECTOORC).

\section{REFERENCES}

[1] L. De Novellis, A. Sorniotti, P. Gruber and A. Pennycott, "Comparison of Feedback Control Techniques for Torque-Vectoring Control of Fully Electric Vehicles," IEEE Transactions on Vehicular Technology, vol. 63, no. 8, pp. 3612-3623, 2014.

[2] T. Goggia, A. Sorniotti, L. De Novellis, A. Ferrara, P. Gruber, J. Theunissen, D. Steenbeke, B. Knauder and J. Zehetner, "Integral Sliding Mode for the Torque-Vectoring Control of Fully Electric Vehicles: Theoretical Design and Experimental Assessment," IEEE Transactions on Vehicular Technology, vol. 64, no. 5, pp. 1701-1715, 2015.

[3] V. Ivanov, D. Savitski and B. Shyrokau, "A Survey of Traction Control and Antilock Braking Systems of Full Electric Vehicles With Individually Controlled Electric Motors," IEEE Transactions on Vehicular Technology, vol. 64, no. 9, pp. 3878-3896, 2015.

[4] H. Fujimoto, J. Amada, and K. Maeda, "Review of traction and braking control for electric vehicle," IEEE Vehicle Power and Propulsion Conference, 2012.

[5] H. Lee and M. Tomizuka, "Adaptive vehicle traction force control for intelligent vehicle highway systems (IVHSs)," IEEE Transactions on Industrial. Electronics, vol. 50, no. 1, pp. 37-47, 2003.

[6] D. Yin, S. Oh and Y. Hori, "A Novel Traction Control for EV Based on Maximum Transmissible Torque Estimation," IEEE Transactions on Industrial Electronics, vol. 56, no. 6, pp. 2086-2094, 2009.

[7] J.S. Young and K.J. Chen, "A Feasible Approach for the Force Control of Traction Wheels Driven by Electric Motors," Asian Journal of Control, vol.18, no. 1, pp. 112-121, 2016. 
[8] T.A. Johansen, I. Petersen, J. Kalkkuhl and J. Ludemann, "Gainscheduled wheel slip control in automotive brake systems," IEEE Transactions on Control Systems Technology, vol. 11, no. 6, pp. 799$811,2003$.

[9] H. Guo, R. Yu, W. Qiang and H. Chen, "Optimal slip based traction control for electric vehicles using feedback linearization," International Conference on Mechatronics and Control, 2014.

[10] H.Z. Li, L. He, M.X. Kang, J. Song, L.Y. Yu and C. Wu, "PID plus fuzzy logic method for torque control in traction control system," International Journal of Automotive Technology, vol. 13, no. 3, pp. 441450, 2012.

[11] F. Jia and Z. Liu, "A LPV traction control approach for independent inwheel electric motor vehicle," $11^{\text {th }}$ World Congress on Intelligent Control and Automation, 2014.

[12] S. Drakunov, U. Özgüner, P. Dix and B. Ashrafi, "ABS control using optimum search via sliding modes," IEEE Transactions on Control Systems Technology, vol. 3, no. 1, pp. 79-85,1995.

[13] M. Amodeo, A. Ferrara, R. Terzaghi and C. Vecchio, "Wheel Slip Control via Second-Order Sliding-Mode Generation," IEEE Transactions on Intelligent Transportation Systems, vol. 11, no.1, pp. 122-131, 2010.

[14] R.D. Castro, R.E. Araujo and D. Freitas, "Wheel Slip Control of EVs Based on Sliding Mode Technique with Conditional Integrators," IEEE Transactions on Industrial Electronics, vol. 60, no. 8, pp. 3256-3271, 2013.

[15] S. Kuntanapreeda, "Super-twisting sliding-mode traction control of vehicles with tractive force observer," Control Engineering Practice, vol. 38, pp. 26-36, 2015.

[16] B. Subudhi and S.S. Ge, "Sliding-Mode-Observer-Based Adaptive Slip Ratio Control for Electric and Hybrid Vehicles," IEEE Transactions on Intelligent Transportation Systems, vol. 13, no. 4, pp. 1617-1626, 2012.

[17] K. Nam, Y. Hori and C. Lee, "Wheel Slip Control for Improving Traction-Ability and Energy Efficiency of a Personal Electric Vehicle," Energies, vol. 8, no. 7, pp. 6820-6840, 2015.

[18] N. M'sirdi, A. Rabhi, L. Fridman, J. Davila, and Y. Delanne, "Second order sliding mode observer for estimation of velocities, wheel slip, radius and stiffness," American Control Conference, 2016.

[19] A. Ferrara and C. Vecchio, "Low vibration vehicle traction control to solve fastest acceleration/deceleration problems via second order sliding modes," American Control Conference, 2007.

[20] L. Yuan, H. Chen, B. Ren and H. Zhao, "Model predictive slip control for electric vehicle with four in-wheel motors," $34^{\text {th }}$ Chinese Control Conference (CCC), 2015.

[21] D.K. Yoo and L. Wang, "Model based wheel slip control via constrained optimal algorithm," IEEE International Conference on Control Applications, 2007.

[22] J. Schmitt and R. Donath, Robert Bosch GmbH, "System for preventing slip of driven wheels," U.S. Patent 5,573,315, 1996.

[23] K. Reif, "Brakes, Brake control and driver assistance systems," vol. 1, Verlag, 2014.

[24] D.D. Hrovat, M.N. Tran and J.L. Yester, Ford Global Technologies, Inc., 1998," Traction control for moving a vehicle from deep snow," U.S. Patent 5,735,362, 1996.

[25] R. Limpert, Brake Design and Safety, SAE International, 2006.

[26] M. Arbitmann, Z. Chen, T. Raste, P. Lauer, M. Muntu and D. Schmitz, "Slip-controlled braking system for electrically driven motor vehicles," U.S. 20140257664 A1, 2014.
[27] E. Giangiulio and D. Arosio, "New validated tire model to be used for ABS and VDC simulations," $3^{\text {rd }}$ International Colloquium on VehicleTyre-Road Interaction, 2006.

[28] G.J. Heydinger, W.R. Garrott and J.P. Chrstos, "The importance of tire lag on simulated transient vehicle response," SAE Technical Paper 910235, 1991.

[29] F. Bottiglione, A. Sorniotti and L. Shead, "The effect of half-shaft torsion dynamics on the performance of a traction control system for electric vehicles," Proceedings of the Institution of Mechanical Engineers, Part D: Journal of Automobile Engineering, vol. 226, no. 9, pp. 1145-1159, 2012.

[30] L.R. Ray, "Nonlinear tire force estimation and road friction identification: Simulation and experiments," Automatica, vol. 33, no. 10, pp. 1819-1833, 1997.

[31] U. Kiencke, "Real time estimation of adhesion characteristic between tyres and road," IFAC World Congress, 1993.

[32] F. Gustafsson, "Slip-based tire-road friction estimation," Automatica, vol. 33, no. 6, pp. 1087-1099, 1997.

[33] C. Canudas De Wit and R. Horowitz, "Observers for tire/road contact friction using only wheel angular velocity information," Conference on Decision and Control, 1999.

[34] J.M. Rodriguez, R. Meneses and J. Orus, "Active vibration control for electric vehicle compliant drivetrains," Annual Conference of the IEEE Industrial Electronics Society, 2013.

[35] H.B. Pacejka, Tire and Vehicle Dynamics, Butterworth Heinemann, 2012.

[36] www.e-vectoorc.eu, last accessed on $8^{\text {th }}$ November 2016.

[37] L. De Novellis, A. Sorniotti, P. Gruber, J. Orus, J.M. Rodriguez, J. Theunissen, J. De Smet, "Direct yaw moment control actuated through electric drivetrains and friction brakes: Theoretical design and experimental assessment," Mechatronics, vol. 26, pp. 1-15, 2015.

[38] L. De Novellis, A. Sorniotti, P. Gruber, "Driving modes for designing the cornering response of fully electric vehicles with multiple motors," Mechanical Systems and Signal Processing, vol. 64-65, pp. 1-15, 2015.

[39] G. Bartolini, "Applications of a sub-optimal discontinuous control algorithm for uncertain second order systems," International Journal of Robust and Nonlinear Control, vol. 7, no. 4, pp. 299-313, 1997.

[40] G. Bartolini, A. Ferrara and E. Usai, "Chattering avoidance by second order sliding mode control," IEEE Transactions on Automatic Control, vol. 43, no. 2, pp. 241-246, 1998.

[41] G. Bartolini, A. Ferrara, A. Pisano, and E. Usai, "On the convergence properties of a 2-sliding control algorithm for non-linear uncertain systems," International Journal of Control, vol. 74, no. 7, pp. 718-731, 2001.

[42] V. Utkin, "Sliding Modes in Control Optimization," Springer-Verlag, 1992.

[43] S. Skogestad and I. Postlethwaite, "Multivariable Feedback Control Analysis and Design," Wiley, 2005.

[44] D. McFarlane and K. Glover, "A loop shaping design procedure using Hœ synthesis," IEEE Transactions on Automatic Control, vol. 37, no. 6, pp. 759-769, 1992.

[45] R.A. Hyde and K. Glover, "The application of scheduled Hळ controllers to a vstol aircraft," IEEE Transactions on Automatic Control, vol. 38, no. 7, pp. 1021-1039, 1993.

[46] Q. Lu, A. Sorniotti, P. Gruber, J. Theunissen and J. De Smet, "Hळ loop shaping for the torque-vectoring control of electric vehicles: Theoretical design and experimental assessment," Mechatronics, vol. 35, 2016, pp. 32-43, 2016. 


\section{APPENDIX}

A. Vehicle parameters

Table IV - Main vehicle parameters

\begin{tabular}{c|c}
\hline \hline Symbol & Value \\
\hline \hline$M$ & $2500[\mathrm{~kg}]$ \\
$C_{d r a g}$ & $2.76\left[\mathrm{~m}^{2}\right]$ \\
$L$ & $0.39[-]$ \\
$J_{w}$ & $2.66[\mathrm{~m}]$ \\
$R_{w}$ & $0.9\left[\mathrm{kgm}^{2}\right]$ \\
$k_{H S}$ & $370[\mathrm{~mm}]$ \\
$i_{\mathrm{s}, 1}$ & $12700[\mathrm{Nm} / \mathrm{rad}]$ \\
$i_{\mathrm{s}, 2}$ & $1 / 4.7[-]$ \\
$T_{\max }$ & $1 / 1.2[-]$ \\
$P_{\max }$ & $200[\mathrm{Nm}]$ \\
$J_{\max }$ & $80[\mathrm{~kW}]$ \\
& $0.016\left[\mathrm{kgm}^{2}\right]$
\end{tabular}

B. Linearized equations for aerodynamic drag resistant and rolling resistance torque

$$
T_{a e r}=\rho C_{d r a g} S R_{w}^{3} \dot{\theta}_{v, 0} \dot{\theta}_{v}-\frac{1}{2} \rho C_{d r a g} S \dot{\theta}_{v, 0}^{2} R_{w}^{3}
$$

For conciseness only $T_{\text {roll,r }}$ is reported.

$$
T_{\text {roll }, r}=f_{0} F_{z, r, 0} R_{w}-f_{2} F_{z, r, 0} R_{w}^{3}\left(i_{g} \dot{\theta}_{m, 0}\right)^{2}+2 f_{2} F_{z, r, 0} R_{w}^{3} i_{g}^{2} \dot{\theta}_{m, 0} \dot{\theta}_{m}
$$

C. State space formulation matrices for Model 4

$$
\begin{gathered}
\dot{x}=A x+B u \\
x=\left\{\dot{\theta}_{m}, \dot{\theta}_{w}, \dot{\theta}_{v}, \theta_{m}, \theta_{w}, T_{m}, T_{d, w, f}\right\}^{T} \\
\tau_{M, r e f} \\
-f_{0} F_{z, f, 0} R_{w} \\
f_{2} F_{z, f, 0} R_{w}^{3}\left(i_{g} \dot{\theta}_{m, 0}\right)^{2} \\
-f_{0} F_{z, r, 0} R_{w} \\
f_{2} F_{z, r, 0} R_{w}^{3}\left(i_{g} \dot{\theta}_{m, 0}\right)^{2} \\
\frac{1}{2} \rho C_{d r a g} S \dot{\theta}_{v, 0}^{2} R_{w}^{3} \\
\frac{\beta_{H S} i_{g}^{2}}{\eta_{g} J_{e q, 3}} \\
\frac{\beta_{H S} i_{g}}{J_{e q, 4}}
\end{gathered}
$$




$$
B=\left[\begin{array}{ccccccc}
0 & 0 & 0 & 0 & 0 & 0 & 0 \\
0 & \frac{1}{J_{e q, 4}} & \frac{1}{J_{e q, 4}} & 0 & 0 & 0 & 0 \\
0 & 0 & 0 & \frac{2}{J_{e q, 5}} & \frac{2}{J_{e q, 5}} & \frac{1}{J_{e q, 5}} & 0 \\
0 & 0 & 0 & 0 & 0 & 0 & 0 \\
0 & 0 & 0 & 0 & 0 & 0 & 0 \\
\frac{1}{\tau_{m}} & 0 & 0 & 0 & 0 & 0 & 0 \\
0 & 0 & 0 & 0 & 0 & 0 & \frac{1}{\tau_{d}}
\end{array}\right]
$$

where:

$$
\begin{gathered}
a_{2,2}=-\frac{\beta_{H S}+2 f_{2} R_{w}^{3} F_{z, f, 0} \dot{\theta}_{w, 0}}{J_{e q, 4}}, a_{3,3}=-\frac{\left(4 f_{2} F_{z, r, 0}+\rho C_{d r a g} S\right) R_{w}^{3} \dot{\theta}_{v, 0}}{J_{e q, 5}} \\
J_{e q, 3}=J_{s, 1}+J_{m}+\frac{i_{s, 1}^{2}}{\eta_{S, 1}} J_{s, 2}+\frac{1}{2} \frac{i_{g}^{2}}{\eta_{g}} J_{H S}, J_{e q, 4}=\frac{1}{2} J_{H S}+J_{w, f}, J_{e q, 5}=2 J_{w, r}+M R_{w}^{2}
\end{gathered}
$$

D. Terms for second order SOSM controller design

$$
\begin{gathered}
\Gamma(x, t)=\frac{v}{J_{e q, 1} i_{g} R_{w} \dot{\theta}_{m}^{2}} \\
\Phi(x, u, t)=-\frac{\ddot{v} \dot{\theta}_{m}-\dot{v} \ddot{\theta}_{m}}{i_{g} R_{w} \dot{\theta}_{m}^{2}}-\frac{\left(\dot{v} F_{w}+v \dot{F}_{w}\right) \dot{\theta}_{m}-2\left(v F_{w}\right) \ddot{\theta}_{m}}{\eta_{g} J_{e q, 1} \dot{\theta}_{m}^{3}}++\frac{1}{J_{e q, 1} i_{g} R_{w}} \frac{\left(\dot{v} T_{m}\right) \dot{\theta}_{m}-2 v T_{m} \ddot{\theta}_{m}}{\dot{\theta}_{m}^{3}}
\end{gathered}
$$

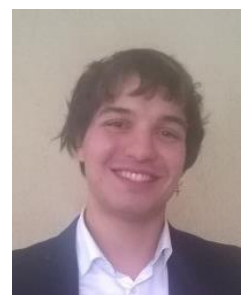

Stefano De Pinto received the M.Sc. degree in mechanical engineering and the Ph.D. degree in mechanical and automotive engineering from the Politecnico di Bari, Bari, Italy, in 2011 and 2015, respectively. Since 2014 he is a Research Fellow in advanced vehicle engineering with the University of Surrey, Guildford, UK. His main research interests are in the areas of hybrid electric vehicles, electric vehicles and vehicle dynamics.

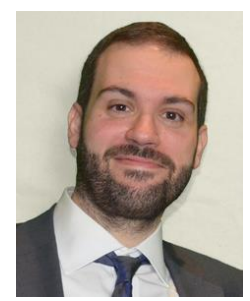

Christoforos Chatzikomis received the M.Sc. degree and the Ph.D. degree in mechanical engineering from the National Technical University of Athens, Greece, in 2003 and 2010, respectively. Since 2015 he is a Research Fellow in electric vehicle control with the University of Surrey, Guildford, UK. His main research interests are in the areas of vehicle dynamics, electric vehicle control and autonomous vehicles.

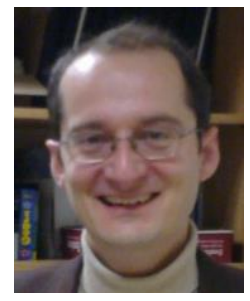

Aldo Sorniotti (M'12) received the M.Sc. degree in mechanical engineering and the Ph.D. degree in applied mechanics from the Politecnico di Torino, Turin, Italy, in 2001 and 2005, respectively. He is a Reader in advanced vehicle engineering with the University of Surrey, Guildford, UK, where he coordinates the Centre for Automotive Engineering. His research interests include vehicle dynamics control and transmission systems for electric and hybrid vehicles.

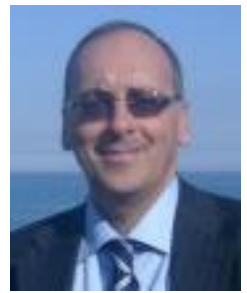

Giacomo Mantriota received the M.Sc. degree in mechanical engineering from the Università di Bari, Bari, Italy, in 1987, and the Ph.D. degree in mechanical engineering from the Politecnico di Bari, Bari, Italy, in 1993. He is a Full Professor in applied mechanics at the Politecnico di Bari. He was Leader of the Industrial Engineering programme (20022012). His research interests are in the areas of automotive, robotics and biomechanics. 